\title{
Erratum to: Clinical Application of a Humanoid Robot in Pediatric Cancer Interventions
}

\author{
Minoo Alemi $^{1,2}$ • Ashkan Ghanbarzadeh ${ }^{1}$ - Ali Meghdari ${ }^{1}$. \\ Leila Jafari Moghadam ${ }^{3}$
}

Published online: 28 October 2016

(C) Springer Science+Business Media Dordrecht 2016

\section{Erratum to: Int J of Soc Robotics \\ DOI 10.1007/s12369-015-0294-y}

A substantial part of this paper was published in volume 8755 of the series Lecture Notes in Computer Science, page 11-22, Title of the book: Social Robotics, 6th International Conference, ICSR 2014, Sydney, NSW, Australia, October 27-29, 2014, ISBN: 978-3-319-11972-4, article title: Impact of a Social Humanoid Robot as a Therapy Assistant in Children Cancer Treatment, by Minoo Alemi, Ali Meghdari, Ashkan Ghanbarzadeh, Leila Jafari Moghadam and Anooshe Ghanbarzadeh.

The authors apologize for not having referenced to the previously published article.

The online version of the original article can be found under doi:10.1007/s12369-015-0294-y.

\footnotetext{
Ali Meghdari

meghdari@sharif.edu

http://meghdari.sharif.edu

Minoo Alemi

alemi@sharif.ir

http://sharif.ir/ alemi/

1 Social Robotics Laboratory, Center of Excellence in Design, Robotics, and Automation Sharif University of Technology, Tehran, Iran

2 Islamic Azad University, Tehran West Branch, Tehran, Iran

3 Mahak Hospital and Rehabilitation Complex, Tehran, Iran
} 